University of Wollongong

Research Online

Australian Institute for Innovative Materials -

Papers

Australian Institute for Innovative Materials

2012

Magnetic and superconducting properties of spin-fluctuation-limited superconducting nanoscale VNx

R Zeng

University of Wollongong, rzeng@uow.edu.au

J Liu

University of Wollongong, jiakunl@uow.edu.au

G Du

Universty of Western Sydney

W. X. Li

University of Wollongong, wenxian@uow.edu.au

J Wang

University of Wollongong

See next page for additional authors

Follow this and additional works at: https://ro.uow.edu.au/aiimpapers

Part of the Engineering Commons, and the Physical Sciences and Mathematics Commons

Research Online is the open access institutional repository for the University of Wollongong. For further information contact the UOW Library: research-pubs@uow.edu.au 


\title{
Magnetic and superconducting properties of spin-fluctuation-limited superconducting nanoscale VNx
}

\author{
Abstract \\ "VNx nanoparticles and nanowires have been prepared by nitrifying V205 nanoparticles (NP) and \\ nanowires (NW). The V205 NP and NW were synthesized by a facile hydrothermal method. Magnetic \\ susceptibility (chi) and magnetization measurements showed long range superconducting ordering \\ (LRSO) at the temperature of $5.8 \mathrm{~K}$ for NW, but there was no observation of LRSO (at least down to $2 \mathrm{~K}$ ) \\ for the NP sample, which is a much lower temperature than for the corresponding bulk, while both NP and \\ $\mathrm{NW}$ showed the absence of long range magnetic ordering, at least down to $2 \mathrm{~K}$. However, the chi data \\ showed that both samples possess a high Pauli-like component, chi(0), in their susceptibility (chi(0) \\ approximate to $2.22 \times 10(-4) \mathrm{emu} / \mathrm{mol}$ for NP and $5 \times 10(-4) \mathrm{emu} / \mathrm{mol}$ for NW). Moreover, for the NW \\ samples, chi has a strong magnetic field dependence and presents a non-linear field-polarization feature, \\ suggesting strong spin-orbit coupling. (C) 2012 American Institute of Physics. [doi:10.1063/1.3679148]"

\section{Keywords} \\ superconducting, properties, spin, fluctuation, limited, nanoscale, magnetic, vnx \\ Disciplines \\ Engineering | Physical Sciences and Mathematics

\section{Publication Details} \\ Zeng, R., Liu, J., Du, G., Li, W., Wang, J., Horvat, J. \& Dou, S. X. (2012). Magnetic and superconducting \\ properties of spin-fluctuation-limited superconducting nanoscale VNx. Journal of Applied Physics, 111 \\ (7), 07E142-1-07E142-4.
}

\section{Authors}

R Zeng, J Liu, G Du, W. X. Li, J Wang, J Horvat, and S X. Dou 


\section{AIP Appilied Physics}

\section{Magnetic and superconducting properties of spin-fluctuation-limited superconducting nanoscale VNx}

R. Zeng, J. Liu, G. D. Du, W. X. Li, J. L. Wang et al.

Citation: J. Appl. Phys. 111, 07E142 (2012); doi: 10.1063/1.3679148

View online: http://dx.doi.org/10.1063/1.3679148

View Table of Contents: http://jap.aip.org/resource/1/JAPIAU/v111/i7

Published by the American Institute of Physics.

\section{Related Articles}

Near-field radiative heat transfer between doped silicon nanowire arrays

Appl. Phys. Lett. 102, 053101 (2013)

Red-green-blue light sensitivity of oxide nanowire transistors for transparent display applications AlP Advances 3, 012112 (2013)

Manganese nanoclusters and MnSi1.7 nanowires formed on Si(110): A comparative x-ray photoelectron spectroscopy study

J. Appl. Phys. 113, 024305 (2013)

Directional-dependent coercivities and magnetization reversal mechanisms in fourfold ferromagnetic systems of varying sizes

J. Appl. Phys. 113, 013901 (2013)

Critical shell thickness for InAs-AlxIn1-xAs(P) core-shell nanowires

J. Appl. Phys. 112, 124305 (2012)

\section{Additional information on J. Appl. Phys.}

Journal Homepage: http://jap.aip.org/

Journal Information: http://jap.aip.org/about/about_the_journal

Top downloads: http://jap.aip.org/features/most_downloaded

Information for Authors: http://jap.aip.org/authors

\section{ADVERTISEMENT}

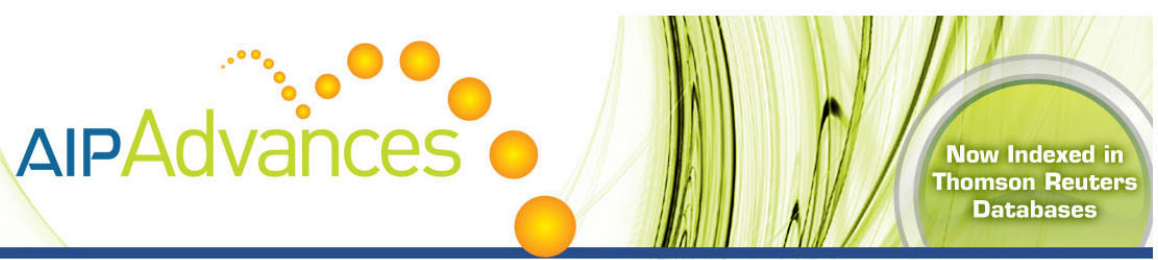

\section{Explore AIP's open access journal: - Rapid publication \\ - Article-level metrics \\ Post-publication rating and commenting}




\title{
Magnetic and superconducting properties of spin-fluctuation-limited superconducting nanoscale $\mathbf{V N}_{\mathbf{x}}$
}

\author{
R. Zeng, ${ }^{\text {a) }}$ J. Liu, G. D. Du, W. X. Li, J. L. Wang, J. Horvat, and S. X. Dou \\ Institute for Superconducting and Electronic Materials, University of Wollongong, NSW 2522, Australia
}

(Presented 3 November 2011; received 11 October 2011; accepted 4 December 2011; published online 12 March 2012)

\begin{abstract}
$\mathrm{VN}_{\mathrm{x}}$ nanoparticles and nanowires have been prepared by nitrifying $\mathrm{V}_{2} \mathrm{O}_{5}$ nanoparticles (NP) and nanowires (NW). The $\mathrm{V}_{2} \mathrm{O}_{5} \mathrm{NP}$ and NW were synthesized by a facile hydrothermal method. Magnetic susceptibility $(\chi)$ and magnetization measurements showed long range superconducting ordering (LRSO) at the temperature of $5.8 \mathrm{~K}$ for NW, but there was no observation of LRSO (at least down to $2 \mathrm{~K}$ ) for the NP sample, which is a much lower temperature than for the corresponding bulk, while both $\mathrm{NP}$ and NW showed the absence of long range magnetic ordering, at least down to $2 \mathrm{~K}$. However, the $\chi$ data showed that both samples possess a high Pauli-like component, $\chi_{0}$, in their susceptibility $\left(\chi_{0} \approx\right.$ $2.22 \times 10^{-4} \mathrm{emu} / \mathrm{mol}$ for NP and $5 \times 10^{-4} \mathrm{emu} / \mathrm{mol}$ for NW). Moreover, for the NW samples, $\chi$ has a strong magnetic field dependence and presents a non-linear field-polarization feature, suggesting strong spin-orbit coupling. ( 92012 American Institute of Physics. [doi:10.1063/1.3679148]
\end{abstract}

The study of nanoscale superconductivity has attracted much attention recently, because it involves such fundamental phenomena as macroscopic quantum tunneling, quantum phase transitions, and environmental effects, and because it has such significant potential applications in electronics circles and quantum computing. ${ }^{1-12}$ One of the key issues is the effect of quantum fluctuations on the dynamic behavior of ultra-small superconductors with dimensions much less than the temperature-dependent coherence length, $\$(T){ }^{1,2}$ Of special interest from both the fundamental and the practical points of view are the dynamical processes of switching between the superconducting and paramagnetic states in the presence of a magnetic field. Small grains can experience spontaneous transitions due to quantum fluctuations. Although experimental studies have been made on a few superconductors, such as MoGe, ${ }^{1,5} \mathrm{~Pb},{ }^{2} \mathrm{Nb},{ }^{5} \mathrm{Al},{ }^{10,11} \mathrm{Sn},{ }^{12}$ and even high temperature superconducting (SC) nanowires ${ }^{13}$ in recent years, there are still controversies on what the expected properties of a onedimensional (1D) superconductor are. For the $\mathrm{VN}_{\mathrm{x}}$ superconductors, one more interesting feature is their spin fluctuation limited superconductivity, which make the superconducting transition temperature $T_{c}<10 \mathrm{~K}$, much lower than the theoretically predicted $\sim 30 \mathrm{~K}^{14-16}$

Achieving fully stoichiometric $\mathrm{VN}_{\mathrm{x}}$ with the $\mathrm{NaCl}$ structure is a difficult proposition. This is because the superconducting transition temperature $\left(T_{c}\right)$ has a linear relationship with the $x$ value. ${ }^{15}$ Single-phase samples have only been successfully prepared with $x$ up to 0.93 and $T_{c}$ up to $9.8 \mathrm{~K}^{14}$ The $\mathrm{N}$ vacancies have dominated the SC $T_{c}$ and other SC properties, and should significantly influence the magnetic properties as well. The $x$ can be determined from $\mathrm{x}$-ray diffraction (XRD) through the lattice parameter and $T_{c}$ measurement results due to the linear variation of $T_{c}$ with $x .{ }^{15}$ To the best of our knowledge, there has been no report yet on the magnetic and superconducting properties of nano-scaled $\mathrm{VN}_{\mathrm{x}}$.

\footnotetext{
a) Author to whom correspondence should be addressed. Electronic mail: rzeng@uow.edu.au.
}

$\mathrm{VN}_{\mathrm{x}}$ nanoparticle (NP) and nanowire (NW) samples were prepared by nitrifying long $\mathrm{V}_{2} \mathrm{O}_{5}$ nanoparticles and nanowires, which had been synthesized by a facile hydrothermal method that will be reported elsewhere.

The XRD patterns of the (220) peak are shown in Fig. 1(a). Analysis indicated that all diffraction peaks can be indexed to a cubic cell with lattice constants $a=b$ $=c=4.112 \AA$ for the NP sample and $a=b=c=4.138 \AA$ for the NW sample, confirming that the crystal structure of the $\mathrm{VN}_{\mathrm{x}}$ belongs to the typical $\mathrm{NaCl}$ structure. The lattice parameter of both samples is smaller than that of fully stoichiometric $\mathrm{VN}_{\mathrm{x}}(x=1)$ due to $\mathrm{N}$ element vacancies. According to the relationship between $x$ and the lattice parameter, ${ }^{15,16}$ we can calculate that $x \approx 0.86$ for NP and $x \approx 0.93$ for NW. The morphology of the $\mathrm{VN}_{\mathrm{x}}$ samples was investigated by SEM, as shown in Fig. 1(b) for NP and Fig. 1(c) for NW. The NP sample clearly has nanoparticles with diameters of $6-10 \mathrm{~nm}$, and the NW sample is shown to have a diameter of $30-80 \mathrm{~nm}$ with a length of a few micrometers. The nanoscale structure generates high surface area, and de-confined free electrons and delocalized spins may appear, so that it would exhibit unique magnetic properties. ${ }^{17}$

The FC $\chi-T$ curve of the NP sample is also shown in the inset of Fig. 2(a). The results show that the superconducting transition temperature $T_{c}=5.8 \mathrm{~K}$ for the NW sample, which is lower than the supposed $T_{c}$, while the superconducting transition cannot be observed for the NP sample down to $2 \mathrm{~K}$. We also plotted the $\chi T$-T curves (Fig. 2(c)) for both the NP and NW samples under different fields and note that the curves show linearly $T$-dependent Pauli-like susceptibility over the $T>T_{c}$ temperature range $(6-300 \mathrm{~K})$. This is because the $\chi-T$ curve can be fit quite well using the sum of a Curie-like susceptibility, $\chi_{C}$, and a linearly $T$-dependent Pauli-like susceptibility, $\chi_{P}$,

$$
\chi=\chi_{C}+\chi_{P}=\left[C_{C W} /\left(T+\Theta_{C W}\right]+\left[\chi_{0}+\chi_{1} T\right],\right.
$$

where $\chi_{0}=4.13 \times 10^{-4} \mathrm{emu} / \mathrm{mol} ; \chi_{1}=-1.68 \times 10^{-7} \mathrm{emu} / \mathrm{mol}$ $\mathrm{K}$; the Curie-Weiss temperature, $\Theta_{C W}=12.8 \mathrm{~K}$; and the Curie constant, $C_{C W}=0.0323 \mathrm{emu} / \mathrm{mol}$, e.g., the effective localized 


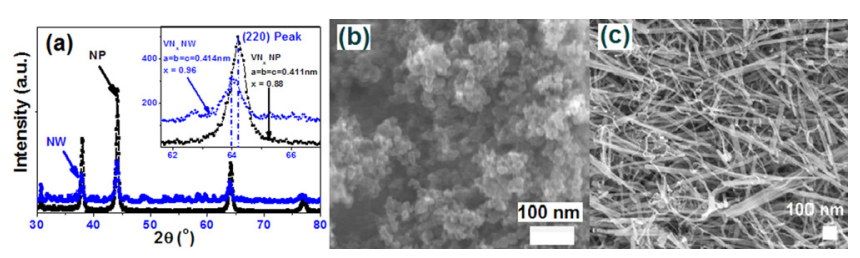

FIG. 1. (Color online) (a) X-ray diffraction patterns of the $\mathrm{VN}_{\mathrm{x}}$ nanoparticles (black symbols) and nanowires (blue symbols). The inset is the pattern of the (220) peaks; the blue dot-dashed lines indicate the (220) peak position of each sample. SEM images of the $\mathrm{VN}_{\mathrm{X}}$ (b) nanoparticles and (c) nanowires.

magnetic moment, $\mu_{\text {eff }}^{\text {Local }}=0.51 \mu_{\mathrm{B}}$ for the NW sample. With similar fitting for the NP sample, we obtained that: $\chi_{0}=2.22 \times 10^{-4} \mathrm{emu} / \mathrm{mol} ; \quad \chi_{1}=-1.13 \times 10^{-7} \mathrm{emu} / \mathrm{mol} \mathrm{K}$; the Curie-Weiss temperature, $\Theta_{C W}=-0.39 \mathrm{~K}$; and the Curie constant, $\quad C_{C W}=0.00455 \mathrm{emu} / \mathrm{mol}$, e.g., $\quad \mu_{\text {eff }}^{\text {Local }}=0.19 \mu_{\mathrm{B}}$. Selected susceptibility $\chi-T$ and $\chi T-T$ curves under different fields are shown in Figs. 2(b) and 2(c), and the fitting results are plotted in Fig. 2(d).

The above fittings indicate that this system presents unusual spin features for the NW sample: (i) There is a large, bare Pauli-like susceptibility $\chi_{0}=4.13 \times 10^{-4} \mathrm{emu} / \mathrm{mol}$, but a small effective magnetic moment $\left(\mu_{\text {eff }}=0.67 \mu_{\mathrm{B}}\right)$, which is much smaller than that of the molecular field theoretical value for spin only $(J=S)$. The $\mathrm{V}^{2+}$ ion local spin moment of $\mu_{\text {eff }}{ }^{J}=S=[4 S(S+1)]^{1 / 2}=3.87 \mu_{B}\left(S=3 / 2\right.$ for the $\mathrm{V}^{2+}$ ion), but this is close to the moment of considering the spinorbit coupling, $\mu_{\text {eff }} J=|L \pm S|=g_{J}[J(J+1)]^{1 / 2}=0.77 \mu_{B} \cdot g_{J}$ is the Lande $g$ factor, while $S$ and $L$ are the spin and orbital quantum numbers, respectively, which suggests that the system has strong spin-orbital coupling. (ii) However, as shown in the inset of Fig. 2(c), the Pauli-like susceptibility contributes strong field dependence, such that $\chi_{0}$ is changed from $4.13 \times 10^{-4} \mathrm{emu} / \mathrm{mol}$ under a field of $1000 \mathrm{Oe}$ to
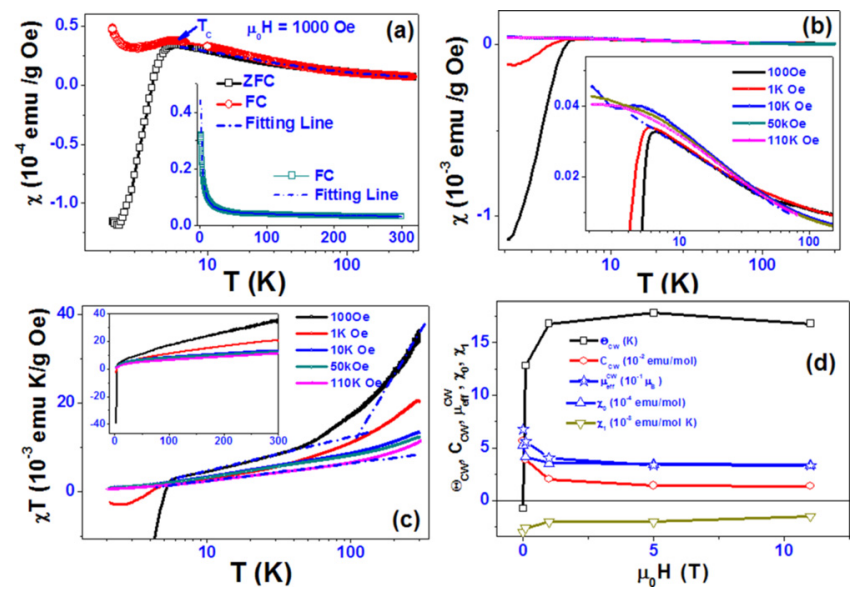

FIG. 2. (Color online). (a) Semi-log plots of $\chi$-T curves under zero field cooling (ZFC: black symbol line) and field cooling (FC: red symbol line) in an external magnetic field $H=1 \mathrm{kOe}$, with the blue dot-dashed line the CurieWeiss fitting line according to Eq. (1) in the text. The inset is the $\chi-T$ curve under an applied $1 \mathrm{~K}$ Oe field for the nanoparticle sample, with the blue dot-dashed line representing the $\chi \propto-\log (T)$ relationship. (b) Semi-log plots of $\chi-T$ curves under the indicated applied fields, with the inset showing an enlargement of the temperature region around $T_{c}$. (c) Semi-log plots of $\chi T-T$ curves under the indicated applied fields; the inset contains normal plots of the $\chi T-T$ curves, with the blue dot-dashed line representing the $\chi T \propto \log (T)$ relationship line. (d) Plots of susceptibility fitting results with field.
$3.37 \times 10^{-4} \mathrm{emu} / \mathrm{mol}$ under a field of $110 \mathrm{kOe}$. Similarly, the effective moment is reduced from $\mu_{\text {eff }}=0.67 \mu_{B}$ to $0.34 \mu_{B}$, which presents a non-linear field-polarization feature, and suggests that the nanostructure with high surface area and high density of defects has delocalized a great amount of electrons, while at the same time, the nanowire 1D structure has confined the electrons, with magnetic field strongly inducing the polarization of free electron spins and spins being localized by strong spin-orbital coupling.

Our susceptibility analysis supports the conjecture that spin-liquid-like behavior may emerge from this system. When the temperature $\mathrm{T}_{\mathrm{c}}<\mathrm{T}<38 \mathrm{~K}$ (100 Oe), $\chi \propto-\log (T)$ (as shown in Fig. 2(b)), or $\chi T \propto \log (T)$ (as shown in Fig. 2(c)), indicating a linear relationship with the log of the temperature, and all the straight lines under different applied fields are nearly parallel to one another, with the field only slightly changing the slopes of the lines.

A series of isothermal magnetization $(M-H)$ loops between 2.1 and $305 \mathrm{~K}$ under fields up to $9 \mathrm{~T}$ has been collected for the NW sample, as shown in Fig. 3. The results include: (a) $M-H$ curves for $T>T_{c}$, (b) $M-H$ curves at temperature $T<$ and near $T_{c}$, (c) decomposition of $M-H$ loops at $T<$ and near $T_{c}$, and (d) critical field $H_{C}-T$ diagram. It is interesting that, at $305 \mathrm{~K}$, the loop shows ferromagnetic-like (FM-like) behavior, which is more clearly shown in the inset of Fig. 3(a), containing the loops after subtraction of the Pauli-like component. Following the suggestion from Eq. (2) that the system can be described in terms of the sum of contributions from the Pauli- and Curie-like terms, we fit the $305 \mathrm{~K}$ magnetization experimental data to:

$$
\begin{aligned}
M(H) & =M_{\text {Pauli }}(H)+M_{\text {Curie }}(H) \\
& =\chi * \times H+M_{0} B\left\{S^{T}, H, T\right\} .
\end{aligned}
$$
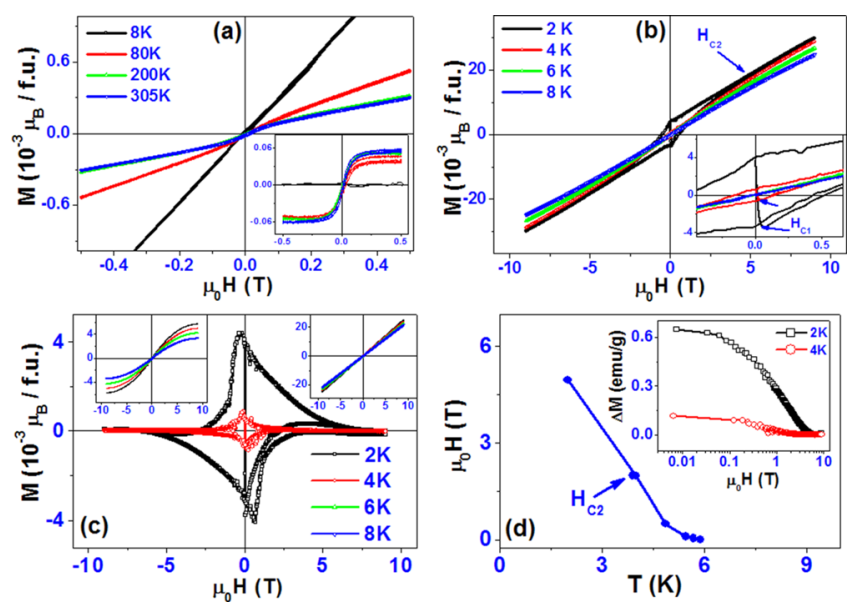

FIG. 3. (Color online). (a) Selected isothermal $M-H$ loops $\left(\mu_{0} H<0.5 \mathrm{~T}\right)$ for $\mathrm{VN}_{\mathrm{x}}$ nanoparticles at $8 \mathrm{~K}, 80 \mathrm{~K}, 200 \mathrm{~K}$, and $305 \mathrm{~K}$, respectively; the inset shows the loops after subtraction of the linear Pauli-like component. (b) $M$ $H$ loops for $\mathrm{VN}_{\mathrm{x}}$ nanoparticles at $8 \mathrm{~K}, 6 \mathrm{~K}, 4 \mathrm{~K}$, and $2 \mathrm{~K}$, respectively; the inset shows the enlarged loops for determining the lower critical field, $H_{c l}$. (c) Spin fluctuation subtracted (superconducting contribution only) isothermal $M-H$ loops for $\mathrm{VN}_{\mathrm{x}}$ nanoparticles at $2 \mathrm{~K}, 4 \mathrm{~K}, 6 \mathrm{~K}, 8 \mathrm{~K}$, and $305 \mathrm{~K}$, respectively; the insets show the straight linear Pauli-component of the loops (right) and the Curie component of the loops with the Pauli-component subtracted (left). (d) Upper critical field $H_{C 2}-T$ curve determined from the $\chi-T$ and $M-H$ curves, with the superconducting critical current density $\left(J_{c}\right)$ related $\Delta M-H$ curves at $2 \mathrm{~K}$ and $4 \mathrm{~K}$ presented in the inset. 
The results can be fitted with the dominant contribution $\chi^{*}=2.14 \times 10^{-4} \mathrm{emu} \mathrm{mol}^{-1} \mathrm{Oe}^{-1}, B\left\{S^{T}, H, T\right\}$ is the Brillouin function, $S^{T}$ is the Stone factor, and $M_{0}=5.27 \times 10^{-3} \mu_{\mathrm{B}} / \mathrm{f}$.u. The behavior of $B\left\{S^{T}, H, T\right\}$ for $H<5 \mathrm{kOe}$ is more typical of a superparamagnet, with $S^{T} \approx 15$. The value of $M_{0}$ corresponds to $5.27 \times 10^{-3} \mu_{\mathrm{B}} /$ f.u., suggesting that only a small number of spins contribute to the second term in Eq. (3). The non-linear low-field magnetization and Curie-like susceptibility seem to arise from a relatively small number of FM spin clusters with $5.27 \times 10^{-3} \mu_{\mathrm{B}} /$ f.u. and $S^{T}=15$. When this is subtracted from $M(H)$ and $\chi^{*}(T)$, we are left with the intrinsic susceptibility of this $1 \mathrm{D}$ system, characterized by a still very large $\chi^{*}=\delta M /$ $\delta \mathrm{H}=2.14 \times 10^{-4} \mathrm{emu} \mathrm{mol}^{-1} \mathrm{Oe}^{-1}$. Similarly, by fitting the $M-H$ loops for temperatures from 6 to $305 \mathrm{~K}$, we obtained that (i) for $305 \mathrm{~K}>T>120 \mathrm{~K}$, the $M_{0}, S^{T}=15$, and $\chi^{*}$ remain nearly constant; (ii) for $120 \mathrm{~K}>T>38 \mathrm{~K}$, the low field $M_{0}$ and $S^{T}$ decrease and $\chi^{*}$ increases with decreasing temperature; an $M_{0} *$ that is polarized in high field appears in this system, and the high-field $M_{0} *$ increases with decreasing $T$; (iii) for $38 \mathrm{~K}>T>6 \mathrm{~K}$, the low field $M_{O}$ disappears, and the high field $M_{0}{ }^{*}, S^{T}$, and $\chi^{*}$ increase with decreasing $T$.

From analysis of the $\mathrm{M}-\mathrm{H}$ loops at temperatures near and below $T_{c}$, as shown in Fig. 2(b), it was found that there is an electron Cooper pair interaction (superconducting state) component added to the system, so that Eq. (2) was modified to the following Eq. (3):

$$
\begin{aligned}
M(H) & =M_{\text {Spin }}(H)+M_{S C}(H) \\
& =\chi * \times H+M_{0} B\left\{S^{T}, H, T\right\}+M_{S C}(H)
\end{aligned}
$$

where the $M_{\text {Spin }}(H)$ is the spin fluctuation contribution, and $M_{S C}(H)$ is the superconducting Cooper pair contribution. We conducted the fitting and subtraction of $M-H$ curves as shown in Fig. 2(b); the $M_{S C}(H)$ component is shown in Fig. 2(c), with the $M_{\text {Curie }}(H)$ and the $M_{\text {Pauli }}(H)$ components shown in the right and left insets of Fig. 2(c), respectively. From the $M_{S C}(H)-H$ loop, we can evaluate the superconducting critical current density $J_{c}$, with $J_{c}$ corresponding to the area of $\Delta M$. The magnetic $J_{c}$ was derived from the height of the magnetization loop $\Delta M$ using the Bean model: $J_{c}=15 \Delta M /[\pi \mathrm{a} 3 \mathrm{~h}]$, where $\mathrm{a}$ and $\mathrm{h}$ are the radius and length of a typical grain in the wire sample. From the superconducting transition temperature point under different fields and the convergence point of the $M_{S C}(H)-H$ loop (as shown in Fig. 3(b)), we can determine the upper field $H_{c 2}-T$ curve, which is plotted in Fig. 3(d), and the $\Delta M-H$ curves at $4 \mathrm{~K}$ and $2 \mathrm{~K}$, which correspond to the $J_{c}$, because it is very hard to determine the size of grains in the nanowire sample, are plotted in the inset of Fig. 3(d). The $J_{c}-H$ curves correspond to the $\Delta M-H$ curves at different temperatures, and the $H_{C 2}-T$ curve presents normal superconducting features, although the $H_{c 2}$ is lower than for the corresponding bulk. ${ }^{14-16}$

It is interesting to notice that coexistence and competition effects of the superconducting Cooper pairs and of the Pauli and field-polarized electrons (Figs. 3(b) and 3(c)) are present in the system, which has motivated us to conjecture the electron pairing and breaking mechanism. Spin fluctuation had been predicted ${ }^{18}$ to exhibit strong effects in $\mathrm{VN}$, which are characterized by a Stoner factor, $S^{T}$, greater than 1 ,

$$
S^{T}=\left(\chi-\chi_{\text {orb }}\right) / \chi_{\text {Pauli }}
$$

where $\chi$ is the susceptibility, $\chi_{\text {Orb }}$ is the orbital susceptibility, and $S^{T}$ represents the exchange enhancement of the nonorbital spin part above the ordinary Pauli term, $\chi_{\text {Pauli }}$. The Pauli-like conduction electrons and electron-phonon coupling are the necessary conditions for the formation of Cooper pairs, which are involved in the spin-orbit coupling in the VN nanowire system. Under lower field, the $\chi_{\text {Pauli }}$ is very large (Fig. 1(d)), and there are even local FM spin clusters. One of the important superconductivity mechanisms of high $T_{c}$ cuprate superconductors is that the Cooper pairs form in a spin liquid induced by antiferromagnetic fluctuations, and this indicates that the emergence of spin liquid behavior is beneficial for the formation of pairs. ${ }^{19}$ It has long been known that magnetic fields suppress superconductivity through two main effects: first, by aligning the electron spins (i.e., the Zeeman effect) and second, by raising the kinetic energy of electrons via Meissner screening currents (i.e., the orbital effect). ${ }^{20}$ For VN, both effects occur to suppress the $T_{c}$, due to strong spin-fluctuation, e.g., Eq. (4) effects. Moreover, in nanosized $\mathrm{VN}_{\mathrm{x}}$, the $\mathrm{N}$ vacancies tend to decrease the $T_{c}$, while nanosized quantum effects further decrease the $T_{C}$, as we obtained $x \approx 0.86$ for NP and $x \approx 0.936$ for NW from the XRD results. These values suggest that $T_{c}$ should be $4.2 \mathrm{~K}$ for $\mathrm{NP}$ and $8.5 \mathrm{~K}$ for $\mathrm{NW}$, much higher than the measured $<2 \mathrm{~K}$ for NP and $5.7 \mathrm{~K}$ for NW.

In summary, the magnetic behavior and the superconducting properties in $\mathrm{VN}_{\mathrm{x}}$ nanoparticles (NP) and nanowires (NW) have been investigated by magnetic measurements. The NP sample with $x=0.86$ exhibits the absence of any long-range spin or superconducting ordering, while the NW sample with $x=0.93$ exhibits long-range superconducting ordering at $5.8 \mathrm{~K}$. Susceptibility measurements indicated that the $\mathrm{VN}_{\mathrm{x}}$ possesses a very high Pauli-like component and suggest that the system exists in a highly spin disordered state. The superconducting ordering suppressed by the $\mathrm{N}$ content $x$, and the particle size and shape, is further suppressed by the strong spin-fluctuation.

The authors thank Dr. T. Silver for her help and useful discussions. This work is supported by the Australian Research Council through a Discovery project (project ID: DP0879070).

\footnotetext{
${ }^{1}$ A. Bezryadin, C. N. Lau, and M. Tinkham, Nature 404, 971 (2000).

${ }^{2}$ Y. Guo, Y. F. Zhang, et al. Science 306, 1915 (2004).

${ }^{3}$ K. A. Matveev, A. I. Larkin, et al. Phys. Rev. Lett. 89, 096802 (2002).

${ }^{4}$ J. Wang, M. Singh, et al. Nat. Phys. 6, 389 (2010).

${ }^{5}$ A. Rogachev and A. Bezryadin, Appl. Phys. Lett. 83, 512 (2003).

${ }^{6}$ J. P. Cleuziou, W. Wernsdorfer, et al. Nat. Nanotechnol. 1, 53 (2006).

${ }^{7}$ K. Y. Arutyunov, Phys. Rep. 464, 1 (2008).

${ }^{8}$ L. Chirolli and G. Burkard, Phys. Rev. B 74, 174510 (2006).

${ }^{9}$ J. G. Wang, M. L. Tian, et al. Nano Lett. 5, 1247 (2005).

${ }^{10}$ M. Zgirski, K.-P. Riikonen, et al. Nano Lett. 5, 1029 (2005).

${ }^{11}$ F. Altomare, A. M. Chang, et al. Phys. Rev. Lett. 97, 017001 (2006).

${ }^{12}$ M. Tian, J. Wang, et al. Appl. Phys. Lett. 83, 1620 (2003).

${ }^{13}$ K. Xu and J. R. Heath, Nano Lett. 8, 3845 (2008).

${ }^{14}$ K. E. Gray, R. T. Kampwrith, et al. Phys. Rev. B 38, 2333 (1988).

${ }^{15}$ F. I. Ajami and R. K. MacCrone, J. Phys. Chem. Solids 36, 7 (1975).

${ }^{16}$ H. L. Luo, S. A. Wolf, et al. Phys. Rev. B 29, 1443 (1984).

${ }^{17}$ J. Liu, et al. Chem. Commun.46, 3887 (2010).

${ }^{18}$ H. Rietschel, H. Winter, et al. Phys. Rev. B 22, 4284 (1980).

${ }^{19}$ P. A. Lee, et al. Rev. Mod. Phys.78, 17 (2006).

${ }^{20} \mathrm{M}$. Tinkham, Introduction to Superconductivity, 2nd ed. (McGraw-Hill, New York, 1996).
} 